Proceedings of the European Conference Physics of Magnetism 2011 (PM'11), Poznań, June 27-July 1, 2011

\title{
Spin-Orbital Liquid on a Triangular Lattice
}

\author{
A.M. Oleś ${ }^{a, b}$ AND J. ChalOUpkA ${ }^{a, c}$ \\ ${ }^{a}$ Max-Planck-Institut fKF, Heisenbergstraße 1, D-70569 Stuttgart, Germany \\ ${ }^{b}$ Marian Smoluchowski Institute of Physics, Jagellonian University, W.S. Reymonta 4, PL-30059 Kraków, Poland \\ ${ }^{c}$ Department of Condensed Matter Physics, Masaryk University, Kotlářská 2, CZ-61137 Brno, Czech Republic

\begin{abstract}
Using Lanczos exact diagonalization of finite clusters we demonstrate that the spin-orbital $d^{1}$ model for triply degenerate $t_{2 g}$ orbitals on a triangular lattice provides an example of a spin-orbital liquid ground state. We also rules, and modify effective spin exchange constants.
\end{abstract} \\ show that the spin-orbital liquid involves entangled valence bond states which violate the Goodenough-Kanamori
}

PACS: 75.10.Kt, 03.65.Ud, 64.70.Tg, 75.10.Jm

\section{Introduction}

A variety of very interesting and challenging problems in condensed matter theory arises in systems of strongly correlated electrons with degenerate orbitals [1]. When the intraorbital Coulomb element $U$ is much larger than the effective electron hopping $t$, i.e., $U \gg t$, the magnetic properties follow from the spin-orbital superexchange [2]. Usually spin interactions are then determined by orbital order and complementary types of spin and orbital order coexist in agreement with the Goodenough-Kanamori rules (GKR) [3]. However, large quantum fluctuations that emerge from strongly frustrated orbital interactions could instead stabilize disordered phases [4]. This observation triggered the search for an example of a spin-orbital liquid (SOL) ground state (GS), similar to a spin liquid state in a one-dimensional Heisenberg antiferromagnet. In principle such a SOL state might be expected for the spin-orbital $d^{9}$ model on a triangular lattice in $\mathrm{LiNiO}_{2}$, but Ising-like orbital interactions suppress it [5].

Exotic behavior of spin-orbital systems follows from spin-orbital entanglement (SOE) [6]. To name a few phenomena, it is responsible for the temperature dependence of optical spectral weights in $\mathrm{LaVO}_{3}$ [7], plays a role in the phase diagram of the $\mathrm{RVO}_{3}$ perovskites [8], and restricts propagation of a hole in states with entangled spin-orbital order [9]. Recently novel phases with SOE were discovered in a bilayer spin-orbital $d^{9}$ model [10], but also here a SOL phase could not be established.

\section{Model and calculation method}

The orbital interactions for $t_{2 \mathrm{~g}}$ orbitals, with $T=1 / 2$ pseudospins that depend on the bond direction, are more quantum than for $e_{\mathrm{g}}$ ones and all three pseudospin components contribute for each bond [1]. Here we employ the Lanczos exact diagonalization to investigate a spin-orbital $d^{1}$ model for triply degenerate $t_{2 \mathrm{~g}}$ orbitals on a triangular lattice as in $\mathrm{NaTiO}_{2}$ [11], with superexchange $\left(\mathcal{H}_{\mathrm{s}}\right)$, direct exchange $\left(\mathcal{H}_{\mathrm{d}}\right)$ and mixed terms responsible for enhanced quantum fluctuations $\left(\mathcal{H}_{\mathrm{m}}\right)$,

$$
\mathcal{H}=J\left[(1-\alpha) \mathcal{H}_{\mathrm{s}}+\sqrt{(1-\alpha) \alpha} \mathcal{H}_{\mathrm{m}}+\alpha \mathcal{H}_{\mathrm{d}}\right] .
$$

The model depends on the parameter $0 \leq \alpha \leq 1$ and on Hund's exchange $\eta \equiv J_{\mathrm{H}} / U$. Here $J$ is the exchange energy. In the direct exchange limit $(\alpha=1)$ the model Eq. (1) is exactly solvable and the GS was determined by considering the valence bond (VB) dimer coverings of the lattice with each dimer containing a spin singlet [12]. Below we show by analyzing the results of the Lanczos diagonalization [13] that a SOL is realized in the opposite superexchange limit $(\alpha=0)$ of the model.

The essential feature of the model Eq. (1) is that $S=1 / 2$ quantum spins are coupled by an SU(2) symmetric $\left(\boldsymbol{S}_{i} \cdot \boldsymbol{S}_{j}\right)$ interactions, while the orbital interactions obey a much lower discrete symmetry between three equivalent directions $\gamma=a, b, c$ in the lattice, with two $t_{2 \mathrm{~g}}$ orbital flavors active in the superexchange via $\left(\boldsymbol{T}_{i} \cdot \boldsymbol{T}_{j}\right)^{(\gamma)}$ term for $T=1 / 2$ pseudospin, and the third one contributing to direct exchange; for more details see [11, 13]. The drastic difference in occupied orbital states realized in both limits at $\eta=0$ is illustrated for the case of a 9-site cluster by insets in Fig. 1b - one finds equal occupancies of each orbital state at $\alpha=0$, while 756 degenerate orbital dimer VB-like states are found at $\alpha=1[12,13]$, and the degeneracy scales with system size.

We characterize the GS by spin, orbital and spin-orbital (four-operator) bond correlations ( $d$ is the degeneracy of the GS manifold $\{|n\rangle\}$ ), given by

$$
\begin{aligned}
\mathcal{S} & \equiv \frac{1}{d} \sum_{n}\left\langle n\left|\boldsymbol{S}_{i} \cdot \boldsymbol{S}_{j}\right| n\right\rangle, \\
\mathcal{T} & \equiv \frac{1}{d} \sum_{n}\left\langle n\left|\left(\boldsymbol{T}_{i} \cdot \boldsymbol{T}_{j}\right)^{(\gamma)}\right| n\right\rangle, \\
\mathcal{C} & \equiv \frac{1}{d} \sum_{n}\left\langle n\left|\left(\boldsymbol{S}_{i} \cdot \boldsymbol{S}_{j}-\mathcal{S}\right)\left(\boldsymbol{T}_{i} \cdot \boldsymbol{T}_{j}-\mathcal{T}\right)^{(\gamma)}\right| n\right\rangle .
\end{aligned}
$$

Below we consider two clusters with periodic boundary conditions (PBC): a hexagonal cluster of $N=7$ sites (N7) and a rhombic cluster of $N=9$ sites (N9). Due to $\mathrm{PBC}$ all sites and bonds are equivalent and the above correlations do not depend on the bond $\langle i j\rangle$ and its di- 

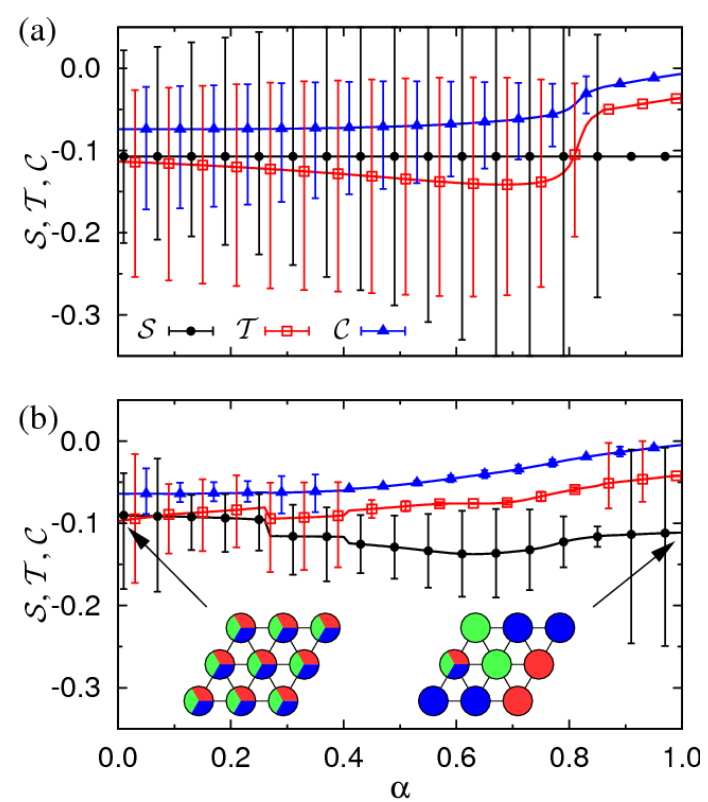

Fig. 1. Bond correlations at $\eta=0$ : $\operatorname{spin}(\mathcal{S})$, orbital $(\mathcal{T})$, and spin-orbital $(\mathcal{C})$ for: (a) N7 cluster, and (b) N9 cluster. The vertical lines indicate the exactly determined range of possible values that follows from the GS degeneracy. The insets in (b) indicate typical orbital patterns in the superexchange $(\alpha=0)$ and direct exchange $(\alpha=1)$ limit for the rhombic N9 cluster.

rection $\gamma$. Each $t_{2 \mathrm{~g}}$ orbital is occupied on average by $1 / 3$ electron, but the states are manifestly different in the limits of $\alpha=0$ and $\alpha=1$, see the insets in Fig. 1b.

\section{Results and discussion}

In both $\mathrm{N} 7$ and $\mathrm{N} 9$ cluster spin $\mathcal{S}$ and orbital $\mathcal{T}$ correlations are negative and the GKR stating that these correlations should be complementary are violated, see Fig. 1. Frustration in the triangular lattice is responsible for a rather weak and independent of $\alpha$ antiferromagnetic (AF) spin correlations in the N7 cluster, $\mathcal{S} \approx-0.107$, see Fig. 1a. These correlations are weaker $(\mathcal{S} \approx-0.090)$ at $\alpha=0$ in the N9 cluster and become more pronounced $(\mathcal{S} \approx-0.144)$ when $\alpha \approx 0.6$ and joint spin-orbital fluctuations weaken to $\mathcal{C} \approx-0.050$, see Fig. 1 b. The orbital correlations weaken as well when $\alpha$ increases toward $\alpha=1$, particularly in the N9 cluster. Joint spin-orbital correlations are similar in both clusters (e.g. $\mathcal{C} \approx-0.070$ at $\alpha=0$ ) and $|\mathcal{C}|$ gradually decreases when spin and orbital operators disentangle approaching $\alpha=1$.

An important question is whether spin order and excitations could be described by an effective spin model derived from Eq. (1). In order to illustrate consequences of SOE in magnetic states we rewrite the $d^{1}$ spin-orbital model Eq. (1) in a general form [2] resembling a spin Hamiltonian,

$$
\mathcal{H}=\sum_{\langle i j\rangle \| \gamma}\left\{\hat{\mathcal{J}}_{i j}^{(\gamma)}\left(\boldsymbol{S}_{i} \cdot \boldsymbol{S}_{j}\right)+\hat{\mathcal{K}}_{i j}^{(\gamma)}\right\},
$$

where the orbital operators $\hat{\mathcal{J}}_{i j}^{(\gamma)}$ and $\hat{\mathcal{K}}_{i j}^{(\gamma)}$ depend on the parameters $\{\alpha, \eta\}$ for a bond $\langle i j\rangle$ along axis $\gamma$. Mean field (MF) procedure used frequently reads [13],

$$
\begin{aligned}
& \mathcal{H}_{\mathrm{MF}}=\sum_{\langle i j\rangle \| \gamma}\left\{\left\langle\hat{\mathcal{J}}_{i j}^{(\gamma)}\right\rangle \boldsymbol{S}_{i} \cdot \boldsymbol{S}_{j}-\left\langle\hat{\mathcal{J}}_{i j}^{(\gamma)}\right\rangle\left\langle\boldsymbol{S}_{i} \cdot \boldsymbol{S}_{j}\right\rangle\right\} \\
& +\sum_{\langle i j\rangle \| \gamma}\left\{\hat{\mathcal{J}}_{i j}^{(\gamma)}\left\langle\boldsymbol{S}_{i} \cdot \boldsymbol{S}_{j}\right\rangle+\hat{\mathcal{K}}_{i j}^{(\gamma)}\right\} .
\end{aligned}
$$

It disentangles spin and orbital degrees of freedom and is used to determine the MF spin constant for N7 and N9 clusters by averaging the orbital operator $\hat{\mathcal{J}}_{i j}^{(\gamma)}$ (its explicit form is given in [13]) over the MF GS $\left|\Phi_{0}\right\rangle$,

$$
J_{\mathrm{MF}} \equiv\left\langle\Phi_{0}\left|\hat{\mathcal{J}}_{i j}^{(\gamma)}\right| \Phi_{0}\right\rangle .
$$

Let us note that the orbital fluctuations in the term $\propto \sqrt{\alpha(1-\alpha)}$ in Eq. (1) contribute here as well as they couple different components of $\left|\Phi_{0}\right\rangle$. In contrast, the exact exchange constant, $J_{\text {exact }}$, is found when the exact GS $|\Phi\rangle$ obtained after Lanczos diagonalization is used.
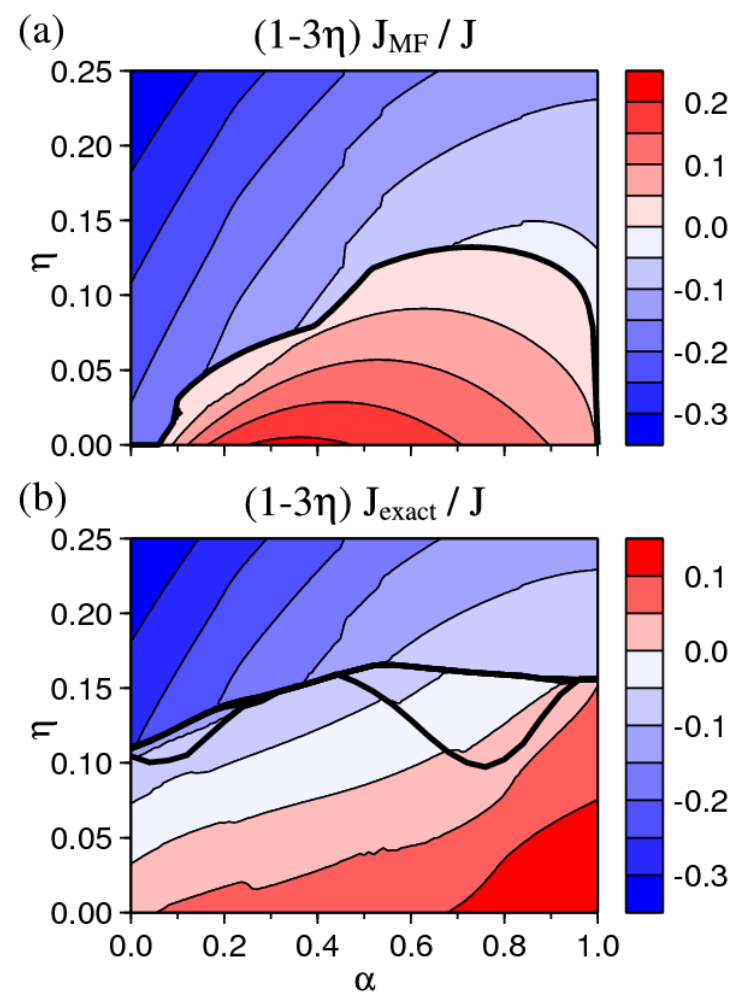

Fig. 2. Phase diagram in the $(\alpha, \eta)$ plane (heavy lines) and exchange constants (contour plots) as obtained for the N9 cluster with PBC: (a) within the MF calculation, and (b) using exact Lanczos diagonalization. In the MF case (a) the transition from low-spin $\left(\mathcal{S}_{t}=1 / 2\right)$ to high-spin $\left(\mathcal{S}_{t}=9 / 2\right)$ phase occurs when $J_{\mathrm{MF}}$ changes sign. In the exact calculation one finds in addition an intermediate phase with $\mathcal{S}_{t}=3 / 2$, stable between the heavy black lines in (b). 
In Fig. 2 we compare the phase diagrams obtained from the above MF procedure and from exact diagonalization for the N9 cluster. Consider first a quantum phase transition from the low-spin $\left(\mathcal{S}_{\mathrm{t}}=1 / 2\right)$ disordered phase to the high-spin $\left(\mathcal{S}_{\mathrm{t}}=9 / 2\right)$ ferromagnetic $(\mathrm{FM})$ phase which occurs for sufficiently large $\eta$. When spin and orbital operators are disentangled in Eq. (6), i.e., $\mathcal{C} \equiv 0$ [6], it coincides with the sign change of the MF exchange constant $J_{\mathrm{MF}}$ and no other phase (with $1 / 2<\mathcal{S}_{\mathrm{t}}<9 / 2$ ) is found, see Fig. 2a, as in a spin system.

Comparing the values of $J_{\mathrm{MF}}$ and $J_{\text {exact }}$ found from the MF and from exact diagonalization of the N9 cluster (Fig. 2), one finds that $J_{\text {exact }} \geq J_{\mathrm{MF}}$ in a broad regime of $\alpha$ except for $\alpha \approx 1$. Therefore, the MF approximation turns out to be rather unrealistic and overestimates (underestimates) the stability of states with FM (AF) spin correlations. The value of $J_{\mathrm{MF}}$ decreases with increasing $\eta$, but positive values $J_{\mathrm{MF}}>0$ are found at $\eta=0$ only if $0.07<\alpha<1$. This demonstrates that FM states: (i) are favored when joint spin-orbital fluctuations are suppressed, and (ii) are stabilized by orbital fluctuations close to $\alpha=0$ even in absence of Hund's exchange. The transition from the low-spin $\left(\mathcal{S}_{\mathrm{t}}=1 / 2\right)$ to the high-spin $\left(\mathcal{S}_{\mathrm{t}}=9 / 2\right)$ state occurs in presence of SOE at a much higher value of $\eta \approx 0.14$, with only weak dependence on $\alpha$, see Fig. 2b. In addition, one finds a phase with an intermediate spin value $\mathcal{S}_{\mathrm{t}}=3 / 2$ for $0<\alpha<0.21$ and $0.44<\alpha<0.88$, and $J_{\text {exact }}$ changes discontinuously at the transition to the FM phase.

Altogether, the qualitative trends found for the N9 cluster are generic and agree with those observed for the N7 cluster, see Fig. 15 in [13]. In both cases one finds that: (i) the FM phase is stable in the MF approximation close to $\alpha=0$ and becomes degenerate with the low-spin phase at $\alpha=1$, (ii) the MF procedure is exact in the regime of FM phase, and (iii) the transition to the FM phase occurs gradually through intermediate values of total spin $\mathcal{S}_{\mathrm{t}}$ (except at $\alpha=1$ ). This suggests that partially polarized FM phase should occur in the thermodynamic limit of the model Eq. (1) and arises due to SOE which is gradually suppressed when $\eta$ increases.

We argue that the present study and the results presented in [13] provide evidence in favor of a quantum SOL phase in the present $d^{1}$ spin-orbital model Eq. (1) in a corner of its phase diagram - in the regime of small values of both $\alpha$ and $\eta$ parameter. In agreement with the directional nature of orbital interactions, this SOL phase is also characterized by rather strong VB dimer correlations [14]. The consequences of SOE are more severe in this phase and the transition to the FM phase occurs typically at a much higher value of $\eta$ than the one where $J_{\text {exact }}$ changes its sign. Therefore, we suggest that even in case when magnetic exchange $J_{\text {exact }}$ is accurately evaluated using the relevant orbital correlations, it loses its predictive power and is inadequate to describe the magnetic ground state and excitations in a system dominated by SOE, where the GKR are violated. Let us note that the frustrated triangular lattice plays here an important role and removes any kind of orbital order.

\section{Summary and conclusions}

Summarizing, we have demonstrated that the GKR are violated in the regime of weak Hund's exchange and the spin-orbital liquid phase is stabilized by spin-orbital entanglement in the $d^{1}$ spin-orbital model on the triangular lattice. In this regime the MF decoupling procedure of spin and orbital operators fails and the magnetic properties can be determined only by solving the full entangled spin-orbital many-body problem.

Finally, we point out that spin-orbital entangled states play a role in exotic ground states [10] as well as low energy excitations for realistic values of $\eta \approx 0.14$. Their consequences have already been established in the vanadium perovskites [7-9], and we expect that they could be of even more importance in systems with geometrically frustrated lattice such as the one considered here.

\section{Acknowledgments}

We thank Bruce Normand for insightful discussions. A.M. Oles acknowledges support by the Foundation for Polish Science (FNP) and by the Polish National Science Center (NCN) project No. N202 069639. J. Chaloupka acknowledges the fellowship of the Alexander von Humboldt Foundation and support by the Ministry of Education of Czech Republic under grant No. MSM0021622410.

\section{References}

[1] A.M. Oleś, Acta Phys. Pol. A 115, 36 (2009).

[2] A.M. Oleś, G. Khaliullin, P. Horsch, L.F. Feiner, Phys. Rev. B 72, 214431 (2005).

[3] J.B. Goodenough, Magnetism and the Chemical Bond, Interscience, New York 1963; J. Kanamori, J. Phys. Chem. Solids 10, 87 (1959).

[4] L.F. Feiner, A.M. Oleś, J. Zaanen, Phys. Rev. Lett. 78, 2799 (1997); J. Phys.: Condens. Matter 10, L555 (1998).

[5] A. Reitsma, L.F. Feiner, A.M. Oleś, New J. Phys. 7, 121 (2005).

[6] A.M. Oleś, P. Horsch, L.F. Feiner, G. Khaliullin, Phys. Rev. Lett. 96, 147205 (2006).

[7] G. Khaliullin, P. Horsch, A.M. Oleś, Phys. Rev. B 70, 195103 (2004).

[8] P. Horsch, A.M. Oleś, L.F. Feiner, G. Khaliullin, Phys. Rev. Lett. 100, 167205 (2008).

[9] K. Wohlfeld, A.M. Oleś, P. Horsch, Phys. Rev. B 79, 224433 (2009).

[10] W. Brzezicki, A.M. Oleś, Phys. Rev. B 83, 214408 (2011); Acta Phys. Pol. A 121, 1045 (2012).

[11] B. Normand, A.M. Oleś, Phys. Rev. B 78, 094427 (2008).

[12] G. Jackeli, D.A. Ivanov, Phys. Rev. B 76, 132407 (2007).

[13] J. Chaloupka, A.M. Oleś, Phys. Rev. B 83, 094406 (2011).

[14] B. Normand, Phys. Rev. B 83, 064413 (2011). 